\title{
A Novel Control Method and Mathematical Model for Intelligent Robot
}

\author{
Nianxiang $\mathrm{Wu}$ \\ Anhui National Defense Vocational College, Anhui, Luan237001, China \\ Received: November 5, 2020. Revised: May 2, 2021. Accepted: May 14, 2021. Published: May 18, 2021.
}

\begin{abstract}
Hamiltonian method based on action micro-control is widely used in the control of mechanical arm synchronous motor. In order to realize the combination of robot dynamics and drive motor control, Hamiltonian control method is used in this paper to exploit a novel controller for robot, which can be used for better steady-state characteristics in the system. However, dynamic response of port-controlled Hamiltonian (PCH) of control system is slower, so the related control method is exploited and coordinated with the proportional-derivative (PD) plus gravity compensation. At this time, the system has both the fast dynamic response of the PD and the steady state of the $\mathrm{PCH}$. The reverse motor method is used and the two controllers are combined by current conversion to realize the overall control of the robot and the drive motor. The robot drive motor is controlled, and the robot joint position control is combined with the drive motor current control by current conversion. It can be seen from the simulation results that the coordinately controlling the end position of robot can reach the desired position quickly and accurately. Moreover, compared with the separate control of PD plus gravity compensation and $\mathrm{PCH}$ control method, it is proved that this scheme has both a fast dynamic process and better performance and ability to resist load torque disturbance. So control method proposed in this paper has a good application prospect.
\end{abstract}

Keywords-Predictive Control, Master Slave Robotic Manipulator, Sliding Mode Controller, Approximation Errors

\section{INTRODUCTION}

A ctuators are required by mechatronic products such as various optical instruments, intelligent control appliances, automated office equipment, vehicle intelligent electronic equipment, industrial robots, precision $\mathrm{CNC}$ machine tools, automatic production lines and automated building safety systems to drive the system up and running. For example, the rotation of the precision CNC machine tool spindle, various movements of the industrial robot control arm, and the feed motion of the spindle table in machining center.

The joint robot whose main form is the industrial robot arm is used in the field of automated assembly. Benefited from its good controllability, simple structure, wide speed range, high accuracy and low noise, permanent magnet synchronous motor (PMSM) is widely used in robotic joint drive systems[1]. Among all the control methods currently applied to robot drive systems, proportional differential (PD) control method is widely used and simple in design, but it is greatly affected by parameters and has poor anti-interference ability, which makes it difficult to be widely used in practical engineering. Lagrangian formula method can derive the general form model of robot dynamics, which effectively solves the dynamic modeling problem of multi-joint robots, and it is suitable for most robot dynamics solving problems [2-3], but the derivation process is relatively troublesome and very difficult to combine with robot drive motor control. Robust adaptive control can effectively solve the uncertainty problem in the system model, and can efficiently track the position and speed of the robot joint, which can correct the law and parameters according to the actual system requirements [4-5]. But its design is complicated. Literature [6] proposes a simple sliding mode control scheme that does not rely on independent stable discontinuous surfaces, which reduces the complexity of the design. A control method of upper bound of interference is introduced in Literature [7], but simple sliding mode control can not eliminate the chattering problem. Design in Literature [8] is based on the feedback linearization control algorithm, which has good control effect, but ignores that the nonlinearized model of linear part will no longer reflect the system characteristics. Neural network adaptive control can approximate dynamic model of the robot, effectively solve the nonlinearity and uncertainty in control system of complex robot, and make the robot effectively track the given trajectory under ideal dynamics with good control performance. Neural network control makes the system has better adaptability and dynamic performance through continuous learning [9-11]. Fuzzy control can be used in combination with other control methods to effectively control complex systems, but fuzzy with simple information has disadvantages of low precision and poor dynamic quality [12-13]. Backstepping method is simple in design and can realize complete decoupling of PMSM control system, which can be used for high-precision motion control of robots [14-15].

\section{EXECUTION DRIVE SYSTEM OF INTELLIGENT ROBOT}

The power supply is supplied to the motor drive system to make the motor move linearly or rotate, then drive the mechanism or load mechanical movement, and stop at the position specified by the controller according to the system 
setting. Power converter is an indispensable intermediate mechanism. There are two types of control motors: linear drive and rotary drive. Open-loop systems do not have the capability to detect performance. Errors cannot be detected and corrections cannot be reached. Such systems are usually driven by stepper motors. When a command pulse is inputted to start, accuracy of the stepper motor is not high. Closed-loop system itself is provided with a detecting device. The system has a feedback signal input and a performance detecting mechanism, which can directly detect the displacement of the moving component. Besides it, a feedback technique and an error compensation technology are adopted by the system so that moving displacement of the moving component can be accurately controlled.

In a semi-closed loop system, detection device mounted on a servo motor detects the displacement and velocity of moving component, which is often an encoder or tachogenerator. Due to the inevitable external factors such as transmission gap and force deformation of the transmission parts, control accuracy of semi-closed loop system is far lower than the accuracy of closed loop system.

Actuator in the electromechanical control system does work on the outside world, namely transmits power which is the necessary to system and relevant motion finally required to achieve the system. Although the power transmitted by the information machine actuator is small, the motion required is high. Generally, the following requirements are met. Actuator can implement various movements set by the system. Basic elements of these system movements should generally include speed, displacement, starting (stop) position, motion trajectory shape and positive and negative directions.

There are also associated accuracy control and sensitivity control requirements for the trajectory and start (stop) points of the motion. Actuator can transmit the power needed for control. It must be considered in terms of the strength and stiffness of the system, and there must be a proper large transmission force or torque. Actuator can guarantee the excellent dynamic quality of entire system. Since motion trajectory and positioning accuracy need to be guaranteed in pressure and high-speed operation, several performance indexes such as actuator's dynamic stiffness, thermal deformation, static stiffness and friction characteristics are required to be considered during the operation of the system.

The intelligent control system converts the input energy into the mechanical energy required by the system through the electronic measurement control device. The way to achieve this function is to send instructions to the transmission mechanism through electronic controller, and the electromechanical transmission mechanism drives the actuator through internal control process to realize energy conversion[16-18].

\section{MATHEMATiCAL Model of INTELLIGENT Robot}

\section{A. Dynamics and Kinematics Model of Robot}

According to the Lagrange equation, dynamics expression of joint robot is

$$
D(q) \ddot{q}+C(q, \dot{q}) \dot{q}+R_{f} \dot{q}+G(q)=\lambda
$$

In Equation 1, $q$ is the n-dimensional displacement vector of joint rotation angular. $\dot{q}$ is the n-dimensional rotational angular velocity vector of the machine joint. $\ddot{q}$ is the ndimensional acceleration vector of the joint rotational angular. $D(q)$ is the symmetrical inertial square matrix. $C(q, \dot{q})$ is centrifugal force matrix. $R_{f}$ is the friction resistance coefficient matrix, and $G(q)$ is the heavy moment vector. $\lambda$ is the input torque vector. Double joint robot motion coordinate axis model is shown in Figure 1.

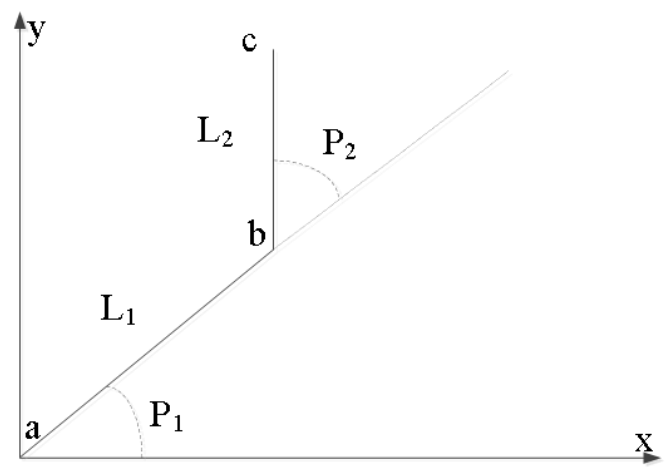

Figure 1. Double joint robot motion coordinate axis model

In Fig. 1, $L_{1}$ and $L_{2}$ respectively represent joint lengths of two robot, and $q_{1}$ and $q_{2}$ are the angular displacements of the two joints[19].

The matrix parameters and vectors in Equation 1 can be used.

$D(q)=\left[\begin{array}{cc}\left(m_{1}+m_{2}\right) L_{1}^{2}+m_{2} L_{2}^{2}+2 m_{2} L_{1} L_{2} & m_{2} L_{2}^{2}+m_{2} L_{1} L_{2} \cos q_{2} \\ m_{2} L_{2}^{2}+m_{2} L_{1} L_{2} \cos q_{2} & m_{2} L_{2}^{2}\end{array}\right]$ $R f=\left(\begin{array}{lr}R_{f 1} & 0 \\ 0 & R_{f 2}\end{array}\right)$

$C(q, \dot{q})=\left[\begin{array}{cc}-m_{2} L_{1} L_{2} \dot{q}_{2} \sin \dot{q}_{2} & -m_{2} L_{1} L_{2}\left(q_{1}+q_{2}\right) \sin q_{2} \\ m_{2} L_{1} L_{2} \dot{q}_{1} \sin q_{2} & 0\end{array}\right]$

$G(q)=\left[\begin{array}{c}\left(m_{1}+m_{2}\right) g L_{1} \sin q_{1}+m_{2} g L_{2} \sin \left(q_{1}+q_{2}\right) \\ m_{2} g L_{2} \sin \left(q_{1}+q_{2}\right)\end{array}\right]$

Where $m_{1}$ and $m_{2}$ respectively represent the mass of two joints.

The relationship two points can be expressed as

$$
{ }_{0}^{2} t={ }_{1}^{0} t{ }_{2}{ }_{2} t=\left[\begin{array}{cccc}
c_{12} & -s_{12} & 0 & L_{1} c_{1}+L_{2} c_{12} \\
s_{12} & c_{12} & 0 & L_{1} s_{1}+L_{2} s_{12} \\
0 & 0 & 1 & 0 \\
0 & 0 & 0 & 1
\end{array}\right]
$$

In Equation 2, $c_{12}=\cos \left(q_{1}+q_{2}\right), \quad s_{12}=\sin \left(q_{1}+q_{2}\right), \quad s_{1}=\sin q_{1}$, $c_{1}=\cos q_{1}$.

According to the position of the robot end $(x d, y d)$ and inverse kinematics of the robot, the angular displacement of the two joints can be separately obtained as[20] 
$\left[\begin{array}{c}q_{1 d} \\ q_{2 d}\end{array}\right]=\left[\begin{array}{c}\arctan \frac{y d}{x d}+\arccos \frac{x_{d}^{2}+y_{d}^{2}+L_{1}^{2}-L_{2}^{2}}{2 L_{1} \sqrt{x_{d}^{2}+y_{d}^{2}}} \\ \arctan \frac{y d}{x d}+\arccos \frac{x_{d}^{2}+y_{d}^{2}+L_{1}^{2}-L_{2}^{2}}{2 L_{1} \sqrt{x_{d}^{2}+y_{d}^{2}}}-\arccos \frac{x_{d}^{2}+y_{d}^{2}-L_{1}^{2}-L_{2}^{2}}{2 L_{1} L_{2}}\end{array}\right]$

\section{B. PMSM Mathematical Model}

The model of PMSM in coordinate system can be expressed as

$$
\left\{\begin{array}{c}
L_{i d} \frac{d i_{i d}}{d t}=-R_{i s} i_{i d}+n_{i p} w_{i} l_{i q} i_{i q}+u_{i d} \\
L_{i q} \frac{d i_{i q}}{d t}=-R_{i s} i_{i q}-n_{i p} w_{i} L_{i d} i_{i d}-n_{i p} w_{i} \phi_{i}+u_{i q} \\
J_{i m} \frac{d w_{i}}{d t}=\lambda_{i}-\lambda_{i L}-\lambda_{i f}=n_{i p}\left[\left(L_{i d}-L_{i q}\right) i_{i d} i_{i q}+\phi_{i} i_{i q}\right]-\lambda_{i L}-\lambda_{i f} \\
\frac{d \theta_{i}}{d t}=w_{i} \\
\lambda_{i}=n_{i p}\left[\left(L_{i d}-L_{i q}\right) i_{i d} i_{i q}+\phi i_{i q}\right] \\
q_{i}=k_{i} \theta_{i}
\end{array}\right.
$$

Where $\mathrm{i}=1,2$. $L, i, u$ and $R$ are respectively the inductance, the current, voltage and resistance on stator. $n_{i p}$ is the pole logarithm. $w_{i}$ is the rotor mechanical angular velocity. $J_{i m}$ is the rotary inertia. $\lambda_{i L}$ is the load torque. $\Phi_{i}$ is the flux linkage for motor. $\theta_{i}$ is the angular displacement for motor. $q_{i}$ is the robot Joint angular displacement.

Given the desired trajectory coordinates of the robot, expected angular displacement of the two joints can be obtained from the inverse kinematics of robot. Robot joint drive torques can be respectively obtained by the robot joint position PCH and PD controller. Coordinated control strategy is designed to obtain the coordinated torque. Then through current transforming, PMSM current is controlled by backstep method to realize the organic combination of the robot and PMSM control.

\section{DESIGN OF Robot Position COORDINATION CONTROL}

\section{A. Position Coordination}

The Hamiltonian function is

$$
H(q, p)=\frac{1}{2} p^{t} d^{-1}(q) p+V(q)
$$

Where $q \in R 2$ and $p \in R 2$ are angular displacement and momentum vector. $V(q)$ is potential energy, and the relationship between $q$ and $p$ can be expressed as

$$
\dot{q}=d^{-1}(q) p
$$

The Hamiltonian model of robot dynamics can be expressed as

$$
\left[\begin{array}{c}
\dot{q} \\
\dot{p}
\end{array}\right]=\left\{\left[\begin{array}{cc}
0 & i_{2} \\
-i_{2} & 0
\end{array}\right]-\left[\begin{array}{cc}
0 & 0 \\
0 & R_{f}
\end{array}\right]\right\}\left[\begin{array}{l}
\frac{\partial H(q, p)}{\partial q} \\
\frac{\partial H(q, p)}{\partial q}
\end{array}\right]+\left[\begin{array}{l}
0 \\
g
\end{array}\right] \lambda_{1}
$$

In Equation 9, $I_{2}$ is a two-dimensional unit matrix. $\lambda_{1}=\left[\lambda_{11}, \lambda_{21}\right]^{t}$ is the input torque vector. Take $g=\operatorname{diag}\{11\}$. Take the expected Hamiltonian function of the robot dynamics system

$$
H d(q, p)=\frac{1}{2} \widetilde{p}^{t} d_{d}^{-1}(q) \widetilde{p}+p \widetilde{q}^{2}
$$

In the above formula, $\widetilde{q}=q-q_{d}, \widetilde{p}=p=p_{d} . p$ is a constant and $D d(q)$ is the desired inertia matrix. At the desired position $\mathrm{q}_{\mathrm{d}}$, there is $p_{d}=0$, and $H_{d}(q, p)$ takes the minimum value. Now there is an input vector $\lambda_{1}$ to make the system meet the desired Closed-loop Hamiltonian control system model.

$$
\left[\begin{array}{c}
\dot{q} \\
\dot{p}
\end{array}\right]=\left[j_{d}(q, p)-R_{d}(q, p)\right]\left[\begin{array}{c}
\frac{\partial H(q, p)}{\partial q} \\
\frac{\partial H(q, p)}{\partial q}
\end{array}\right]
$$

Take anti-symmetric matrix

$$
\begin{aligned}
& j d=-j_{d}^{t}=\left[\begin{array}{cc}
0 & d^{-1}(q) D_{d}(q) \\
-D_{d}(q) D^{-1}(q) & J_{2}(q, p)
\end{array}\right], \\
& R d=R_{d}^{t}=\left[\begin{array}{cc}
0 & 0 \\
0 & g K_{v} g^{t}
\end{array}\right],
\end{aligned}
$$

Where $K_{v}=K_{v}^{t}>0$. It can be obtained from equations (9) and (11) that

$$
\begin{aligned}
& \left\{\left[\begin{array}{cc}
0 & i_{2} \\
-i_{2} & 0
\end{array}\right]-\left[\begin{array}{cc}
0 & 0 \\
0 & R_{f}
\end{array}\right]\right\}\left[\begin{array}{l}
\frac{\partial H(q, p)}{\partial q} \\
\frac{\partial H(q, p)}{\partial q}
\end{array}\right]+\left[\begin{array}{l}
0 \\
g
\end{array}\right] \lambda_{1}= \\
& \left\{\left[\begin{array}{cc}
0 & D^{-1}(q) D_{d}(q) \\
-D_{d}(q) D^{-1}(q) & j_{2}(q, p)
\end{array}\right]-\left[\begin{array}{cc}
0 & 0 \\
0 & g K_{v} g^{t}
\end{array}\right]\right\}\left[\begin{array}{l}
\frac{\partial H(q, p)}{\partial q} \\
\frac{\partial H(q, p)}{\partial q}
\end{array}\right]
\end{aligned}
$$

Select the desired Hamiltonian function as the Lyapunov function, namely $V 1=H d(x), x=[q, p]^{t}$, then the $\mathrm{PCH}$ controller can be obtained from equation (12)

$$
\left[\begin{array}{c}
\lambda_{11} \\
\lambda_{21}
\end{array}\right]=\left[\begin{array}{l}
\frac{\partial H(q, p)}{\partial q}-R_{f} \frac{\partial H(q, p)}{\partial p}-D_{d} D^{-1} \frac{\partial H_{d}(q, p)}{\partial q} \\
+\left(J_{2}(q, p)-g K_{v} g^{t}\right) \frac{\partial H_{d}(q, p)}{\partial p}
\end{array}\right]
$$




$$
\left[\begin{array}{c}
\lambda_{11} \\
\lambda_{21}
\end{array}\right]=\left[\begin{array}{c}
\left(m_{1}+m_{2}\right) g L_{1} \sin q_{1}+m_{2} g L_{2} \sin \left(q_{1}+q_{2}\right) \\
-m_{2} L_{1} L_{2} \dot{q}_{1} \dot{q}_{2} \sin \dot{q}_{2}-m_{2} L_{1} L_{2}\left(q_{1}+q_{2}\right) \dot{q}_{2} \sin q_{2} \\
-R_{f 1} \dot{q}_{1}=K_{v 1}\left(\dot{q}_{1}-\dot{q}_{1 d}\right)-2 p\left(q_{1}-q_{1 d}\right) \\
m_{2} g L_{2} \sin \left(q_{1}+q_{2}\right) m_{2} L_{1} L_{2} \dot{q}_{1} \sin q_{2}+R_{f 2} \dot{q}_{2} \\
-K_{v 2}\left(\dot{q}_{2}-\dot{q}_{2 d}\right)-2 p\left(q_{2}-q_{2 d}\right)
\end{array}\right]
$$

The PD plus gravity compensation control law can be expressed as

$$
\lambda_{2}=-K_{p} \widetilde{q}-K_{d} \dot{\widetilde{q}}+\hat{G}(q)
$$

where $\lambda_{2}=\left[\lambda_{12}, \lambda_{22}\right]^{t}$ is input torque vector.

$K_{p}=\operatorname{diag}\left(K_{p 1} \quad K_{p 2}\right)$ is scale factor matrix.

$K_{d}=\operatorname{diag}\left(K_{d 1} \quad K_{d 2}\right)$ is differential coefficient matrix.

$\hat{G}(q)$ is heavy torque estimate. Take $\hat{G}(q)=G(q)$. Dynamics equation of robot is

$$
D(q) \ddot{q}+\left[C(q, \dot{q})+K_{d}\right] \dot{q}+R_{f} \dot{q}+K_{p} q=0
$$

The PD plus gravity compensation controller can be obtained as follows.

$$
\left[\begin{array}{c}
\lambda_{12} \\
\lambda_{22}
\end{array}\right]=\left[\begin{array}{c}
-k_{p 1}\left(q_{1}-q_{1 d}\right)-k_{d 1}\left(\dot{q}_{1}-\dot{q}_{1 d}\right) \\
+\left(m_{1}+m_{2}\right) g L_{1} \sin q_{1}+m_{2} g L_{2} \sin \left(q_{1}+q_{2}\right) \\
-K_{p 2}\left(q_{2}-q_{2 d}\right)-K_{d 2}\left(\dot{q}_{2}-\dot{q}_{2 d}\right)+m_{2} g L_{2} \sin \left(q_{1}+q_{2}\right)
\end{array}\right]
$$

Construct a coordination function.

$$
c_{1}(t)=\frac{1}{2}+\frac{1}{2} \tanh \left(\frac{1}{2}(t-b)\right), c_{2}(t)=\frac{1}{2}+\frac{1}{2} \tanh \left(\frac{1}{a}(t-b)\right)
$$

Where $C l(t)$ and $C 2(t)$ are the coordination parameters of the PCH and PD plus gravity compensation controllers. $a$ and $b$ are constants. The robot joint position controller is obtained separately, and design coordinated control scheme.

\section{B. Continuous Motion}

According to robot joint motion flow in single operation process, the control timing designed for the continuous motion is divided into automatic reciprocating starting condition, robot entering the circulating logic state, robot completion and fault recovery state and so on.

(1) Logic design of automatic reciprocating star.

Step 1 : After the continuous instruction is completed, "Service Call" service request signal is issued.

Step 2: Robot requests to start. "Machine Request Start" joint stop request signal turns OFF, and "Joint start request" joint start request signal turns $\mathrm{ON}$. After robot continuous command is fully executed, "Machinecontinuouscommand" joint stop detection signal turns OFF, "Jointstopdetection" joint continuous command signal turns $\mathrm{ON}$, and "Jointcontinuouscommand" robot allows the enter signal to turn ON.
Step 3: Robot turns OFF the "Robotarmup-stop" the robot interference signal and then goes into standby.

(2) Robot joint work

Step1: Robot moves to chuck position of spindle 1 when the gripper clamps the processed workpiece, and then turns the "HD1 select" spindle 1 selection signal to $\mathrm{ON}$, and waits for the motion feedback "HD1selectcheck" spindle 1 to select the feedback signal ON. Robot turns "Chuckclose" clamp request signal OFF. "Chuckopen" release request signal turns ON, and waits for in-position chuck to release. After it, "Chuckclosecheck" chuck clamp detection OFF, then "Chuckopencheck" chuck release detection ON.

Step2: The robotic claw grasps the processed workpiece, then moves the workpiece out of the chuck to open area, and rotates the claw at a certain angle so that the claw holding the unprocessed workpiece faces the chuck.

Step3: Robot inserts the unprocessed workpiece into the chuck, then turns "Chuckopen" release request signal OFF. "Chuckclose" clamp request signal turns ON, then waits for the chuck to clamp the workpiece, and "Chuckopencheck" chuck releases detection OFF . After it, "Chuckclosecheck" chuck clamps detection $\mathrm{ON}$.

(3) Robot exits the continuous command

Step1: Robot releases the unprocessed workpiece, and moves to stop zone. Finally turn "Robot armup-stop"the robot interference signal ON.

Step2: Robot requests to turn off the sensor. "Request to turn off the sensor" sensor off signal is turned OFF, and "Sensor closed" sensor off completion signal turns ON. After sensor is completely turned off, "Joint sensing closure" shutdown sensing signal turns OFF.

(4) Robot to be started.

Robot sends "Robot Be Activated" service completion pulse signal to the terminal. The joint servo motor turns "Joint Servomotor" service request signal OFF and then stops the program.

\section{Joint Movement}

For the operation of the robot joint, the PNP point-to-point signal whose number is large is adopted. Ethernet/IP industrial bus is used in the system proposed in this paper to connect major peripherals, and connect safety loop with point-to-point IO, which simplifies wiring, and makes it easy to install, commission and maintain. In terms of operation, the touch screen panel not only saves the hardware such as the button indicator, but also concentrates the data of the entire system on the touch screen for convenient observation and maintenance. The electrical architecture is shown in Figure 2. 


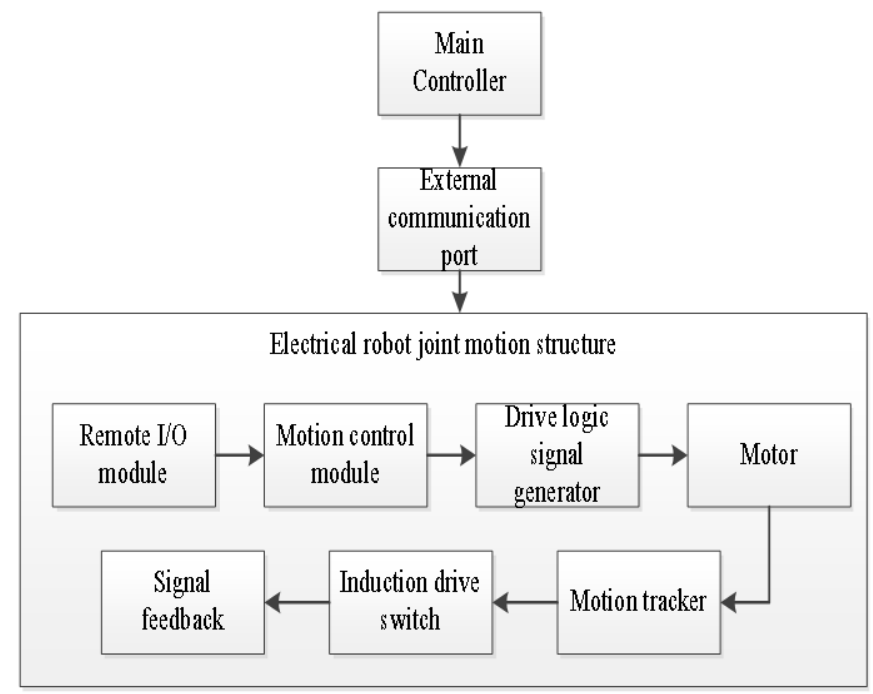

Figure 2. Robot Electrical Architecture

The work process is as follows.

Step1: Manually input position, action and target information. Step2: Send data to robot signal receiving end, and start system. Step3: Robot senses various parameter settings and target conditions, and sets the driving signal.

Step4: Send a driving signal to the driving logic switch, and check the shutdown motion track.

Step5: Robot performs trajectory operation from the target ground to realize all processes of positioning, moving, grabbing, moving, and action.

Step6: Robot confirms whether the instruction is completed, if there is a next instruction, and finally returns the joint to 0 position.

\section{SIMULATION}

\section{A. Hardware}

The hardware mainly includes motor control drive circuit, motor angle feedback circuit and CAN communication. The motor control driving circuit includes PWM output of controller, power amplification of PWM by IR2104, and finally driving $\mathrm{H}$-bridge circuit. The motor angle feedback circuit is mainly the connection between the peripheral circuit of AS5045 chip and the controller pin to realize the real-time reading of magnetic encoder position by DsPIC30F / 4012. CAN communication is mainly the peripheral interface circuit of can driver chip MCP2551. Through this chip, DsPIC30F / 4012 and USBCAN2 are connected in series into the same can network to realize the data reception and transmission between dsPIC30F / 4012 and PC.

The single joint servo control system takes DsPIC30F / 4012 digital signal controller and AS5045 magnetic rotation absolute value encoder as the core. It is mainly composed of DSC, H-bridge driver chip, H-bridge circuit, position signal feedback circuit and can bus communication interface.DSC and AS5045 realize closed-loop control of the motor, and PC realizes can communication through USBCAN2 and DSC.
AS5045 is a contactless magnetic rotary encoder, which is used for accurate measurement of $360^{\circ}$ angle in the range. The resolution of absolute angle measurement is $0.0879^{\circ}$, three hundred and $360^{\circ}$ corresponding to 4096 locations. This product is a system on chip, which integrates integrated Hall components, analog front end and data signal processing functions in a single package. The position sensor can be formed by placing a bipolar rotating magnet above the center of the chip. The magnet can be arranged above or below the IC, as shown in Figure 3.

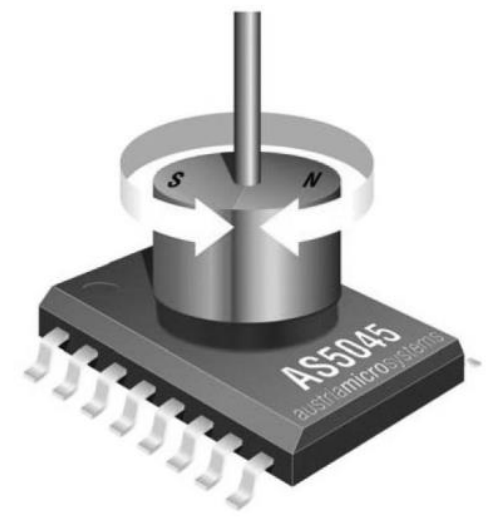

Figure 3. AS5045 and magnet placement

AS5045 is a non-contact magnetic rotary encoder. It uses a rotating current hall technology to detect the magnetic field distributed on the chip surface, and provides user programmable zero position setting and diagnosis. Embedded with DSP engine, it can detect the direction of the magnetic field and calculate the 12 bit binary code, which can be accessed through the synchronous serial interface (SSI). In addition, the absolute angle value can be indicated by pulse width modulation (PWM) signal. The peripheral circuit is shown in Figure 4.

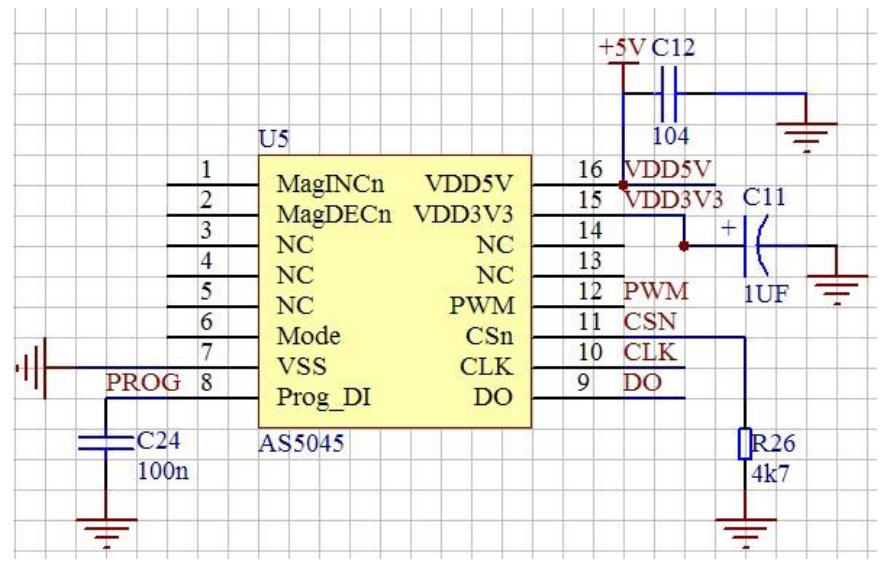

Figure 4. AS5045 data feedback circuit

DsPIC30F / 4012 controller integrates can module and supports can1.2, can2.0A, CAN2.0B passive and CAN2.0B active versions. As long as the CAN bus transceiver MCP2551 is added, the data frame can be sent and received. The peripheral interface circuit of mcp2551 is shown in Figure 5. 


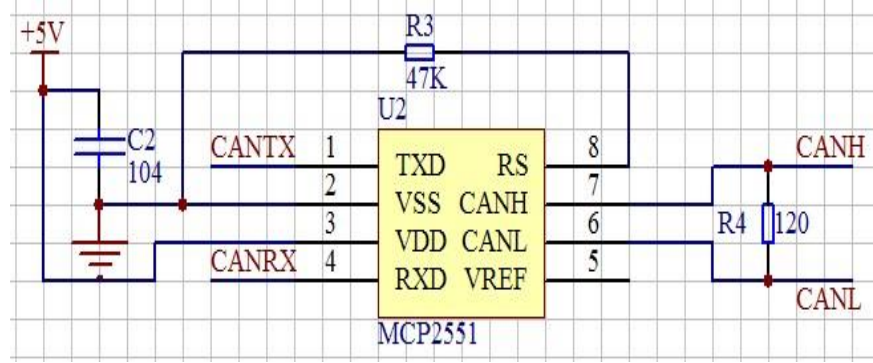

Figure 5. CAN interface circuit

The joint control board PCB and the real object are shown in Figure 6 and Figure 7.

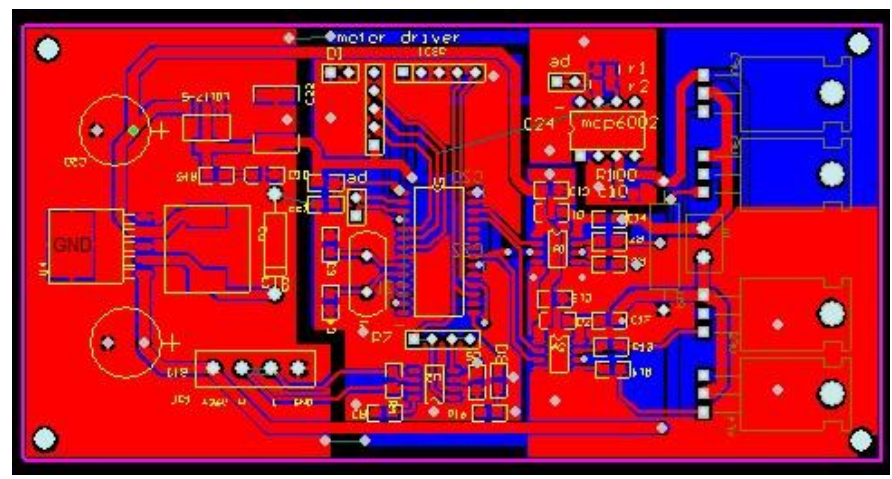

Figure 6. PCB drawing of joint control board

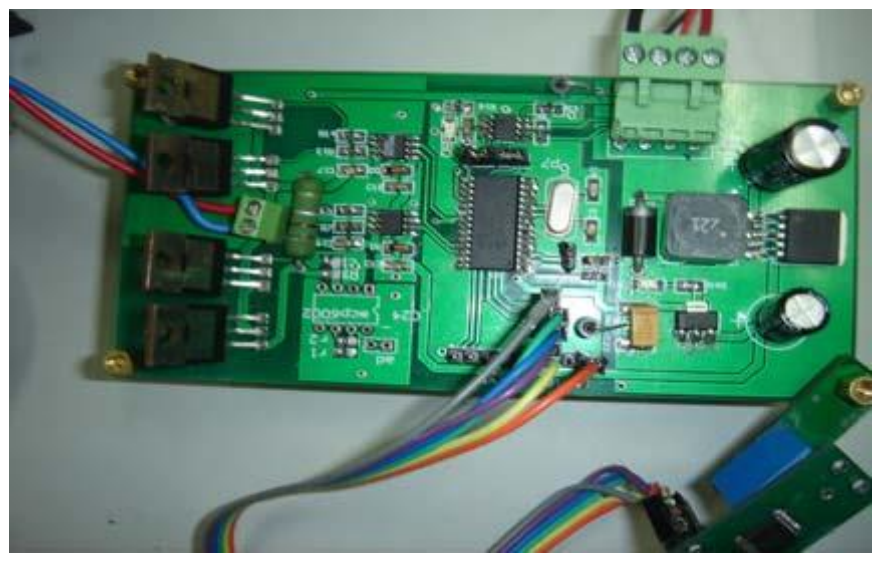

Figure 7. Joint control board

\section{B. Characteristics of System Load}

Taking Lyapunov function into whole system, the robot joint position PCH control system and the PMSM current control system are asymptotically stable. Robot parameters are shown in Table 1 and Table 2.

Table 1. Robot parameter settings

\begin{tabular}{cc}
\hline Setting item & Set value \\
\hline $\mathrm{L} 1=\mathrm{L} 2$ & $1 \mathrm{~m}$ \\
$\mathrm{~m} 1=\mathrm{m} 2$ & $1 \mathrm{~kg}$ \\
$\mathrm{Rf}$ & $\operatorname{diag}\{(2.10 .3)\}$ \\
$\mathrm{Kv}$ & $\operatorname{diag}\{(10.756)\}$ \\
$\mathrm{Kp}$ & $\operatorname{diag}\{20100)\}$
\end{tabular}

$\mathrm{Kd}$

$\left.\operatorname{diag}\left\{\begin{array}{ll}3 & 10\end{array}\right)\right\}$

Table 2. PMSM parameters

\begin{tabular}{cc}
\hline Setting item & Set value \\
\hline $\mathrm{L}_{\mathrm{id}}=\mathrm{L}_{\mathrm{iq}}$ & $0.0085 \mathrm{H}$ \\
$\mathrm{R}_{\mathrm{is}}$ & $2.875 \Omega$ \\
$\mathrm{n}_{\mathrm{ip}}$ & 4 \\
$\Phi_{\mathrm{i}}$ & $0.175 \mathrm{~Wb}$ \\
$\mathrm{~J}_{\text {im }}$ & $0.02 \mathrm{~kg} / \mathrm{m}^{2}$ \\
$\mathrm{k}_{1 \mathrm{~d}}=\mathrm{k}_{2 \mathrm{~d}}=\mathrm{k}_{1 \mathrm{q}}=\mathrm{k}_{2 \mathrm{q}}$ & 10000 \\
$\mathrm{a}$ & 0.2 \\
$\mathrm{~b}$ & 0.5
\end{tabular}

When the end position of the robot moves from point (1.5, 0.5 ) to point $(0.5,1.5)$, and $\mathrm{t}=4 \mathrm{~s}$, the system load torque changes as shown in Fig. 3 and Fig. 4.

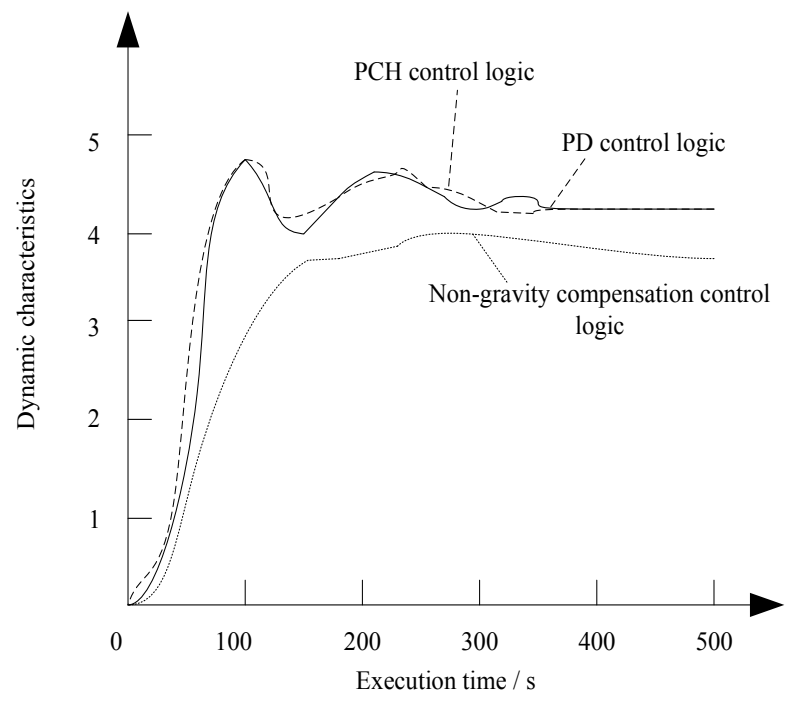

Figure 8. System load torque dynamics

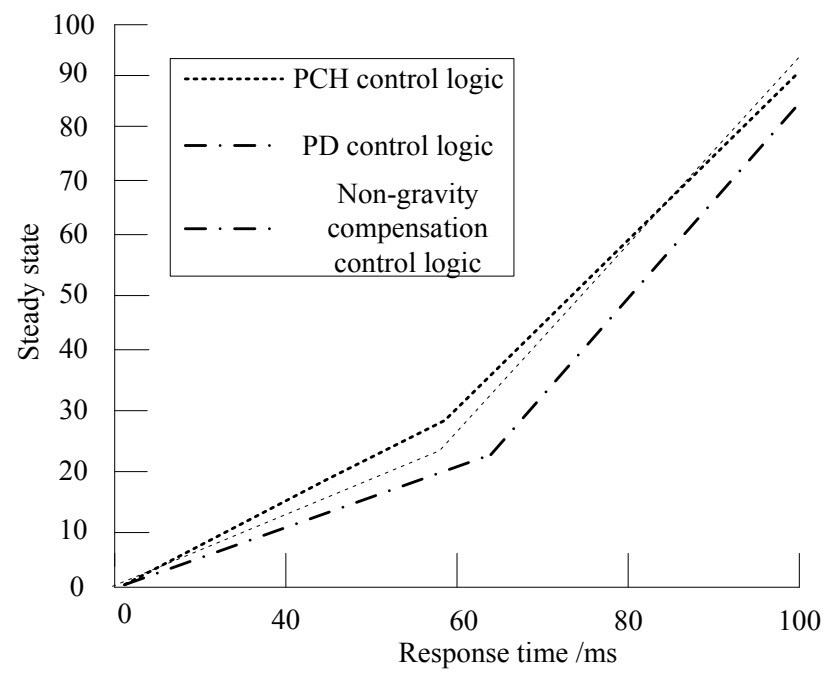

Figure 9. System load steady state characteristics 
It can be seen from Fig. 8 and Fig. 9 that coordinated control can combine the advantages of the two control methods well. With the increase of load torque, when the direction of joint motion is opposite to the direction of external torque $(0900 \mathrm{~h} \sim$ $04 \mathrm{ffh}$ ), the error is negative, but the control accuracy remains at $0.18^{\circ}$. When the direction of joint motion is the same as the external moment of force, the moment of inertia and dynamic torque of the joint increase, so the joint motion speed increases, and the system is affected by Coriolis acceleration, and the error is positive; The steady-state accuracy is $180^{\circ}$ The error is $0.27^{\circ}$. Because the motor in this position is loaded the most, and the joint movement direction is the same as the load torque direction, the overshoot is large, and the steady-state error is the largest. The experimental response time of the system is longer than the simulation time, because the actual system is a highly nonlinear and uncertain system, while the simulation ignores some influence factors, such as friction, motor viscosity, and does not consider the influence of assembly errors such as the coaxiality of joint assembly on the system. In the experiment, because the movement direction is the same as the external torque direction, it is easy to cause overshoot when the movement speed is fast, but the system quickly adjusts to a stable state. From the maximum overshoot and steady-state accuracy, it can be seen that sliding mode control can still achieve high-precision control and good dynamic quality in the case of load.

\section{CONCLUSION}

This paper mainly discusses the related execution drive technology in modular robots, and comprehensively discusses the performance characteristics of PCH and PD coordinated control. The requirements of the electromechanical control system actuators are discussed and studied. As for the design of motion model, it's the design of the conversion energy between the electronic control device and the actuator interface. The input energy is converted into the mechanical energy required by the system through the electronic measuring control device. The way to realize this function is to send instructions to the transmission mechanism through the electronic controller, and the electromechanical transmission mechanism drives the actuator through the internal control process to realize the energy conversion. Theoretical calculation method for the selection of one-dimensional working platform motor is summarized in this paper. The research method has a good generalization.

Based on the analysis of the existing stage results, through the comparison with other research methods in the same field, combined with the problems still existing in practical application, the mobile robot positioning uncertainty processing proposed in this paper needs to be further improved. The algorithm of perception and location in clutter dynamic environment. In the non-static environment, there are few dynamic obstacles, so it is relatively simple to detect and filter dynamic obstacles, data association, tracking and positioning. However, in the chaotic dynamic environment, the complexity of mobile robot environment perception and positioning is greatly increased. Future research should study how to improve the accuracy and real-time of dynamic obstacle detection and filtering, so as to improve the accuracy of the static model of the environment to achieve the accurate positioning of mobile robot.

\section{REFERENCES}

[1] Wajiansyah A, Supriadi S. Implementasi Master-slave pada Embedded system menggunakan komunikasi RS485. ELKHA, 2020, 12, pp. 26-37.

[2] Barnett E, Gosselin C. Large-Scale 3D Printing With A CableSuspended Robot. Additive Manufacturing, 2015, 7, 27-44.

[3] Bento M . Design of a wide-area damping controller to tolerate permanent communication failure and time delay uncertainties. Energy Systems, 2021, 3, pp. 65-77.

[4] Wu T, Li Q, Bao X B, et al. Time-delay signature concealment in chaotic secure communication system combining optical intensity with phase feedback. Optics Communications, 2020, 475:126042.

[5] Wen S, Guo G . Sampled-Data Control for Connected Vehicles With Markovian Switching Topologies and Communication Delay. IEEE Transactions on Intelligent Transportation Systems, 2020, 21, pp. 29302942.

[6] Chung H, Ma Q, Sayginer M, et al. A Packaged 0.01-26-GHz SingleChip SiGe Reflectometer for Two-Port Vector Network Analyzers. IEEE Transactions on Microwave Theory and Techniques, 2020, PP(99):1-15.

[7] Tapar J, Kishen S, Emani N K. Spectral singularities and asymmetric light scattering in PT-symmetric 2D nanoantenna arrays. Optics Letters, 2020, 45, pp. 375-382.

[8] GK Mertin, M Oldenburger, E Richter, Revised theory of entropy and reversible energy flow in galvanic cells. Journal of Power Sources, 2021, 482:228813.

[9] Van Den Berg J, Abbeel P, Goldberg K. LQG-MP: Optimized path planning for robots with motion uncertainty and imperfect state information. The International Journal of Robotics Research, 2019, 30(7): 895-913.

[10] Badmos T A, Omolaye P O, Mebawondu J, et al. Robot Path Planning Performance Evaluation of a Dynamic Environment. IOSR Journal of Electronics and Communication Engineering, 2020, 13, pp.19-26..

[11] Atia M G B, El-Hussieny H, Salah O. A Supervisory-Based Collaborative Obstacle-Guided Path Refinement Algorithm for Path Planning in Wide Terrains. IEEE Access, 2020,170: 257-266.

[12] Ould Mohamed Mohamed Vall, Modeling and Networked Control of Two-rigid Link Robot Arm, WSEAS Transactions on Systems and Control, Volume 15, 2020, Art. \#39, pp. 375-382.

[13] Lucjan Setlak, Rafal Kowalik, Dynamics of the Designed Robotic Manipulator in the CAD Program, WSEAS Transactions on Applied and Theoretical Mechanics, Volume 14, 2019, Art. \#7, pp. 66-74.

[14] Aboutalebian B , Talebi H A , Etedali S, et al. Adaptive control of teleoperation system based on nonlinear disturbance observer ScienceDirect. European Journal of Control, 2020, 53, pp. 109-116.

[15] Ishiguro Y, Makabe T , Nagamatsu Y, et al. Bilateral humanoid teleoperation system using whole-body exoskeleton cockpit TABLIS. IEEE Robotics and Automation Letters, 2020, 99, 122-138.

[16] Islam M R , Protik P, Das S, et al. Mobile robot path planning with obstacle avoidance using chemical reaction optimization. Soft Computing, 2021,10, pp.1-28.

[17] Jung J W , Park J S , Kang T W , et al. Mobile Robot Path Planning Using a Laser Range Finder for Environments with Transparent Obstacles. Applied Sciences, 2020, 10, pp.2799.

[18] Martyshkin A I . Motion Planning Algorithm for a Mobile Robot with a Smart Machine Vision System. Nexo Revista Científica, 2021, 33, pp.651-671.

[19] Fethi, Matoui, Boumedyen, et al. Contribution to the path planning of a multi-robot system: centralized architecture. Intelligent Service Robotics, 2020, 13(1), pp. 147-158.

[20] Shih B Y, Chang H, Chen C Y. RETRACTED: Path planning for autonomous robots-a comprehensive analysis by a greedy algorithm. Journal of Vibration and Control, 2019, 19(1): 130-142. 


\section{Creative Commons Attribution License 4.0} (Attribution 4.0 International, CC BY 4.0)

This article is published under the terms of the Creative Commons Attribution License 4.0

https://creativecommons.org/licenses/by/4.0/deed.en_US 